\title{
Dark initial state radiation and the kinetic mixing portal
}

\section{Thomas G. Rizzo}

SLAC National Accelerator Laboratory, 2575 Sand Hill Rd., Menlo Park, CA, 94025 U.S.A.

E-mail: rizzo@slac.stanford.edu

Abstract: Data from Planck measurements of the cosmic microwave background (CMB) place important constraints on models with light dark matter (DM) and light mediators especially when both lie in the mass range below $\sim 1 \mathrm{GeV}$. In models involving kinetic mixing where the dark photon acts as the mediator, these constraints are easily satisfied and the appropriate DM relic density achievable if the DM is, e.g., a complex scalar, where $p$-wave annihilation occurs, or is the lighter component of a split pseudo-Dirac state where co-annihilation dominates. In both of these cases, although higher order in the dark gauge coupling, $g_{D}$, the corresponding annihilation processes including dark photon initial state radiation (ISR) will be dominantly $s$-wave with essentially temperature independent cross sections. The rates for these dark ISR associated processes, though not yielding cross sections large enough to contribute to the relic density, can still run into possible conflicts with the bounds arising from the CMB. In this paper we perform a preliminary study of the present and potential future constraints that the CMB imposes on the parameter spaces for both of these scenarios due to the existence of this dark ISR. Further analyses of the effects of dark ISR in DM annihilation is clearly warranted.

Keywords: Beyond Standard Model, Cosmology of Theories beyond the SM

ARXIV EPRINT: 2006.08502 


\section{Contents}

1 Introduction 1

2 General model features and the complex scalar DM scenario 4

2.1 Basic setup 4

2.2 Complex scalar DM scenario 5

3 Pseudo-Dirac fermion DM scenario 11

4 Discussion and summary $\quad 16$

\section{Introduction}

The nature of dark Matter (DM) and its possible interactions with the particles of the Standard Model (SM) other than via gravity remains a great mystery. While Weakly Interacting Massive Particles (WIMPs) [1,2] and axions [3-5] remain as quite viable contenders for the role of DM, increasingly sensitive experiments have failed to yield any convincing signals [6-8] for these long anticipated states. The continued shrinking of the allowed parameter spaces for these scenarios has stimulated a rapid growth of new DM models covering exceedingly wide ranges in both possible masses and couplings [9-12]. As current experiments seemingly disfavor the interaction of DM with us via SM strength couplings, other new interactions must likely exist to explain, e.g., how DM reaches its observed relic abundance $[13,14]$. One way to classify such new interactions is via a set of 'portals' linking the SM with fields in the dark sector. Only a few renormalizable, dimension-4 portals exists; of these, the vector boson/kinetic mixing (KM) portal has received a wide amount of attention in the literature ([15-20]; there has been a huge amount of work on this subject, see for example [21-29], for a general overview and introduction to this framework see [30]) and will be the subject of our discussion below. In the simplest version of such a scenario, the DM fields are SM singlets but are instead charged under a new $\mathrm{U}(1)_{D}$ gauge interaction, with corresponding gauge coupling $g_{D}$, mediated by a dark photon (DP) [31] whose mass can be generated by the dark analog of the SM Higgs mechanism. This DP then experiences $\mathrm{KM}$ with the $\mathrm{SM} \mathrm{U}(1)_{Y}$ hypercharge gauge boson at, e.g., the 1-loop level via a set of 'portal matter' fields which are charged under both gauge groups [32-34]. After the gauge fields are canonically normalized and the usual SM and dark spontaneous symmetry breakings occur, the DP picks up a small coupling to the SM fields. For a DP in the mass range below a few $\mathrm{GeV}$, this coupling is quite well approximated simply by $\simeq \epsilon e Q_{e m}$, where $\epsilon$ is a small dimensionless parameter, $\sim 10^{-(3-4)}$ in the present discussion, that describes the magnitude of this loop-suppressed KM. 
The KM scenario is of special interest when the DM and DP are both relatively light $\lesssim 1 \mathrm{GeV}$ as in such a case the DM can be a thermal relic in a manner similar to what occurs in the WIMP scenario. ${ }^{1}$ For such a range of masses, pair annihilation of DM to achieve the proper relic density via the exchange of a virtual DP usually results in pairs of electrons, muons, or light charged hadrons. For DM at the $\sim 10-1000 \mathrm{MeV}$ mass scale, ${ }^{2}$ constraints from Planck [40] on the CMB tell us that at $z \sim 10^{3}$ the DM annihilation cross section into light charged states, e.g., $e^{+} e^{-}$, must be relatively suppressed [41-43] to avoid injecting additional electromagnetic energy into the plasma. ${ }^{3}$ This constraint lies roughly [47] at the level of $\sim 5 \times 10^{-29}\left(m_{\mathrm{DM}} / 100 \mathrm{MeV}\right) \mathrm{cm}^{3} \mathrm{~s}^{-1}$, and is seen to depend approximately linearly on the DM mass so that it becomes relatively inconsequential above masses $\gtrsim 10-30 \mathrm{GeV}$. Further it is to be noted that this constraint is expected to improve by roughly a factor of $\sim 2-3$ in the coming years [48-50]. This bound for DM annihilation in this $\sim 10-1000 \mathrm{MeV}$ mass range, however, lies several orders of magnitude below the cross section required to reach the observed relic density during freeze out, e.g., $<\sigma v_{\text {rel }}>_{F O} \simeq 4.4(7.5) \times 10^{-26}$ $\mathrm{cm}^{3} \mathrm{~s}^{-1}$ for an $s$-wave annihilating, self-conjugate fermion (or a $p$-wave annihilating complex scalar) DM $[13,14]$. This requirement puts a strong constraint on the nature of the DM interacting with the SM via the DP as well as on their relative masses. For example, if $m_{\mathrm{DM}}>m_{\mathrm{DP}}$, then DM can annihilate into a pair of DPs which will likely be the dominant mechanism to achieve the observed relic density. As an $s$-wave process, the cross section for this reaction during freeze out and during the time of the CMB would naturally be quite similar and so this reaction, as well as those for other $s$-wave annihilation processes, would necessarily be forbidden for light DM by the above mentioned constraints. Clearly a set of mechanisms that reduce the DM annihilation cross section as the temperature, $T$, decreases are needed for DM and DP in this mass range.

One obvious way to avoid this constraint is to require that $m_{\mathrm{DM}}<m_{\mathrm{DP}}$ and also that the $s$-channel DP-mediated annihilation process be $p$-wave so that it is velocity-squared suppressed at times much later than freeze out; this is quite helpful since $v_{\text {rel }}^{2} \sim T$. For spin1 mediators such as the DP, this possibility restricts the DM to be a Majorana fermion ${ }^{4}$ or a complex scalar, $\phi$. Note that since Dirac fermion DM annihilation via an $s$-channel, spin1 exchange with only vector couplings is dominantly $s$-wave, this possibility is forbidden within the above range of masses. A second scenario is that the DM is pseudo-Dirac, forming two mass eigenstates, $\chi_{1,2}$, that are split in mass and which co-annihilate to the $\mathrm{SM}$ via the DP. For a fixed mass splitting, as the temperature drops this co-annihilation process becomes highly Boltzmann suppressed thus avoiding the CMB constraints. The parameter spaces of these two classes of models for light DM/DP in the mass range of interest to us here have been widely studied $[10,11]$ and it is known that the observed

\footnotetext{
${ }^{1}$ The DP and DM masses are naturally similar in several scenarios, e.g., models with extra dimensions where the scale of their masses is set by the (inverse) size of the compactification scale, $R$. See, e.g., refs. [35-38].

${ }^{2}$ The rough lower bound on the DM mass of $\sim 10 \mathrm{MeV}$ is taken from ref. [39].

${ }^{3}$ Important constraints of a very similar magnitude also arise from data from Voyager $2[44,45]$.

${ }^{4}$ The Majorana fermion DM has only have axial-vector couplings to the DP while the DP simultaneously would have vector-like couplings to the SM. This is not realized within the present setup without more complexity in the dark sector, e.g., additional fermions to cancel gauge anomalies.
} 

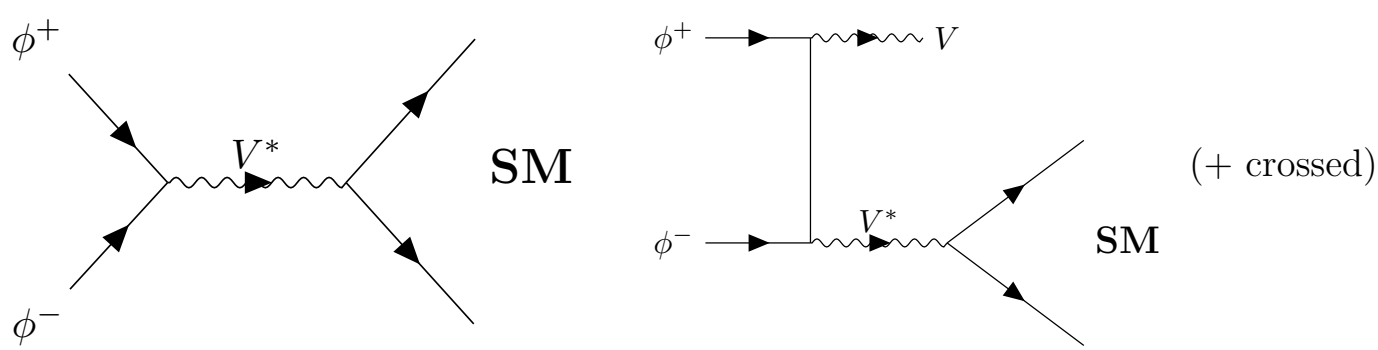

Figure 1. The two basic processes of interest to us in this paper symbolically employing the case of complex scalar DM as a representative example. (Left) DM annihilation into SM final states via $s$-channel DP exchange. (Right) The same process with an additional DP emitted from the initial state producing $u$ - and $t$-channel graphs. For the case of complex scalars the 4-point vertex graph (not shown) is also present.

relic density is achievable in both of these setups while still satisfying all other existing experimental constraints.

For SM singlet DM, the lifting of velocity (and/or helicity) suppression of DM annihilation in many models via the final state radiation (FSR) of SM gauge bosons off of other $\mathrm{SM}$ fields in the final state is well-known [51-53]. In our mass range of interest, the only possible on-shell FSR would be via photons being radiated off of, e.g., an $e^{+} e^{-}, \mu^{+} \mu^{-}$or light charged hadron final state. Less well studied, but of similar, and in some cases more, importance is the initial state radiation (ISR) of the dark mediator field, here the DP, off of the annihilating DM [54]. Note that since the DM can only be a SM singlet in the present scenario, such ISR must necessarily be dark at tree-level. Given the discussion above, this process can obviously be of most relevance to us when $m_{\mathrm{DM}}<m_{\mathrm{DP}}<2 m_{\mathrm{DM}}$ which is a relatively narrow mass window but is of important experimental interest since within it any DPs produced on-shell can decay only to SM final states. There are many existing and proposed searches for such classes of events [55-63]. Figure 1, for the case of complex scalar DM, shows the usual annihilation mechanism via the $s$-channel exchange of a DP in the left panel while the analogous process with additional dark ISR is shown symbolically in the right panel. Note that in the specific case of complex scalar DM, the 4-point process also contributes besides the $t$ - and $u$-channel graphs while such a contribution is absent for the case of pseudo-Dirac DM.

We note, and as will be examined further below, that dark ISR can, in principle, lead to some affects which are not possible via FSR: $(i)$ In the case of complex scalar DM, FSR cannot lift the $p$-wave suppression since this is the result of the (non-relativistic) coupling of the two spin-0, on-shell initial states with the spin-1, off-shell DP. This is easy to see as in the non-relativistic limit, the usual scalar coupling, $\left(p_{1}-p_{2}\right)_{\mu}$ has, to $O\left(v_{\text {rel }}^{2}\right)$, only non-zero 3 -spatial components, $\simeq m_{\mathrm{DM}} v_{\text {rel }}$, so that this automatically leads to a $p$-wave process. Dark ISR changes this situation in that now one of the DM particles in the initial state annihilating via the virtual DP, itself goes off-shell. We now make a rather simple observation: although this process is both $\sim g_{D}^{2} / 4 \pi=\alpha_{D}$ and 3-body phase space suppressed, it is still dominantly an $s$-wave annihilation mechanism, though it is too small to make any significant contribution to the DM relic density. However, 
it can yield a rate which is sufficiently large so as to be in potential conflict with the CMB constraints above and thus lead to restrictions on the model parameter space; we will examine this possibility below. (ii) Similarly, in the case of pseudo-Dirac DM, the two DM mass eigenstates couple in an off-diagonal manner to the DP while the 'diagonal' couplings, which would be unsuppressed $s$-wave annihilations, are absent at lowest order in $g_{D}$. It is well known that obtaining the observed relic density via the co-annihilation process requires that the relative mass splitting of these two eigenstates be relatively small, i.e., $\delta=\Delta m / m_{\mathrm{DM}} \ll 1$. If not, this process becomes highly Boltzmann suppressed by a factor of order $\sim e^{-\delta x_{f}}$ with $x_{f}=m_{\mathrm{DM}} / T_{F O} \simeq 20-30$. As we will see below, dark ISR may partially negate this (very) large Boltzmann suppression even in cases where this mass splitting is no longer so small, i.e., $\delta \lesssim 0.25$, but at the cost of this additional power of $\sim \alpha_{D}$ and 3-body phase space. The resulting rate is, however, too small to make a significant contribution to the relic density. But in this case too, an $s$-wave process is the net result so that the question again arises as to whether or not the bounds from the CMB remain satisfied once ISR of a DP occurs. We will address this issue within this model context in the subsequent analysis as well. Clearly the implications of ISR dark radiation for the allowed parameter space of a given DM model warrants some further examination and that is goal of the preliminary analysis below.

The outline of this paper is as follows: in section 2, we will discuss the general overarching model structure that we will consider and then immediately turn our attention to the specific case of complex scalar DM. In particular, we will explore the extent to which dark ISR can lead to conflicts with the constraints arising from the CMB and the corresponding restrictions this imposes on the complex DM model parameter space. Similarly, in section 3, we will explore the impact of dark ISR in the case of pseudo-Dirac DM wherein co-annihilation is the dominant process leading to the observed relic density. We again will examine the possible conflict with the CMB constraints that can arise and which lead to restrictions on the model parameter space even in cases where the mass splitting between the two eigenstates is significant and contrast this with the previously examined case of complex scalar DM. A discussion and our conclusions can be found in section 4 .

\section{General model features and the complex scalar DM scenario}

We first briefly summarize the common features of the basic framework that we consider below, restate our essential assumptions and then establish subsequent notation before continuing with our analysis.

\subsection{Basic setup}

The interactions in the (mostly) dark gauge/Higgs sector of our model in the original weak eigenstate basis are described by the general Lagrangian

$$
\begin{aligned}
L= & -\frac{1}{4} \hat{V}_{\mu \nu} \hat{V}^{\mu \nu}-\frac{1}{4} \hat{B}_{\mu \nu} \hat{B}^{\mu \nu}+\frac{\epsilon}{2 c_{w}} \hat{V}_{\mu \nu} \hat{B}^{\mu \nu} \\
& +\left(D_{\mu} S\right)^{\dagger}\left(D^{\mu} S\right)-U\left(S^{\dagger} S\right)-\lambda_{H S} H^{\dagger} H S^{\dagger} S+L_{\mathrm{SM}},
\end{aligned}
$$


where $\hat{V}, \hat{B}$ are the kinetically mixed $\mathrm{U}(1)_{D} \mathrm{DP}$ and the SM weak $\mathrm{U}(1)_{Y}$ hypercharge gauge fields, respectively, with the strength of this KM being described by the dimensionless parameter $\epsilon$; here $c_{w}=\cos \theta_{w}$ with $\theta_{w}$ being the usual SM weak mixing angle. Since we will assume that the KM parameter is very small in what follows, $\epsilon \simeq 10^{-(3-4)}$, except where necessary we can work to leading order in $\epsilon$. In this limit the KM is removed by the simplified field redefinitions $\hat{B} \rightarrow B+\frac{\epsilon}{c_{w}} V, \hat{V} \rightarrow V . H, S$ denote the SM and dark Higgs fields, respectively, while $L_{\mathrm{SM}}$ describes the rest of the interactions of the SM. The vacuum expectation value of $S, v_{s} / \sqrt{2}$, resulting from minimizing the potential $U$, generates the mass of the DP, $m_{\mathrm{DP}}=m_{V}=g_{D} Q_{D}(S) v_{s}$, via the dark covariant derivative $D_{\mu}=\partial_{\mu}+i g_{D} Q_{D}(S) \hat{V}_{\mu}$. Here, $g_{D}$ is the $\mathrm{U}(1)_{D}$ gauge coupling and $Q_{D}(S)$ is the relevant dark charge of $S$. Due to the vevs of both $H$ and $S, \lambda_{H S} \neq 0$ generates a mass mixing between the SM and dark Higgs fields which we will necessarily assume to be very small to avoid the strong bounds arising from invisible Higgs decays [64] and so will be considered to be phenomenologically irrelevant in the subsequent discussion. It will also be assumed that $m_{S}>(1-2) m_{V}$, similar to what happens in the SM, so that $S$ is guaranteed to be unstable and can decay sufficiently rapidly as, e.g., $S \rightarrow 2 V$ (or $\rightarrow V V^{*}$ depending on the mass ratio) although the specific details of this will not be required for the discussion below.

\subsection{Complex scalar DM scenario}

While the form of $L$ above is quite general, the addition of the DM itself will introduce some new terms to this set of interactions. In this subsection, we consider the case where the DM is a complex scalar, $\phi$; the form of the general additional pieces of the Lagrangian in this case are given by

$$
L_{\mathrm{DM}}=\left(D_{\mu} \phi\right)^{\dagger}\left(D^{\mu} \phi\right)-U_{\phi}\left(\phi^{\dagger} \phi\right)-\left(\lambda_{H \phi} H^{\dagger} H+\lambda_{\phi S} S^{\dagger} S\right) \phi^{\dagger} \phi
$$

where $D_{\mu}$ is the relevant covariant derivative for $\phi$, whose dark charge will be taken to be unity in what follows without loss of generality. The potential, $U_{\phi}$, describes the (taken to be weak) DM self-interactions which we assume do not generate a vev for $\phi$ so that it can remain stable. The quartic couplings of $\phi$ with both $S$ and $H$, described by the coefficients $\lambda_{\phi(S, H)}$, respectively, will also be assumed to be quite small and to play no essential role in what follows.

Under this set of assumptions, the DM-DP gauge interaction completely dominates and leads to a slightly modified version of the familiar expression from, e.g., ref. [65] for the annihilation cross section of scalar DM pairs into SM fields via the process in the left panel of figure 1 in our mass range of interest; summing over electron, muon and light charged hadronic final states, this is given by

$$
\sigma_{\mathrm{DM}} v_{\mathrm{rel}}=\frac{g_{D}^{2} \epsilon^{2} e^{2}}{6 \pi} \frac{s \beta_{\phi}^{2}}{\left(s-m_{V}^{2}\right)^{2}+\left(\Gamma_{V} m_{V}\right)^{2}}\left[1+\frac{\beta_{\mu}\left(3-\beta_{\mu}^{2}\right)}{2} \theta\left(s-4 m_{\mu}^{2}\right)+R \theta\left(s-4 m_{\pi}^{2}\right)\right],
$$

where $s$ is the usual center of mass energy, $\beta_{\phi, \mu}^{2}=1-4 m_{\phi, \mu}^{2} / s$ are the squares of the DM (muon) velocities, $\Gamma_{V}$ is the total decay width of the DP, and $R$ is the familiar cross section ratio $R=\sigma\left(e^{+} e^{-} \rightarrow\right.$ hadrons $) / \sigma\left(e^{+} e^{-} \rightarrow \mu^{+} \mu^{-}\right)$via virtual photon exchange for 
massless muons. Of course, this hadronic contribution only turns on above the two-pion threshold, $\sim 280 \mathrm{MeV}$; note that the electron mass has been neglected in the expression for $\sigma_{\mathrm{DM}} v_{\text {rel }}$. Here we observe the explicit $\beta_{\phi}^{2}$-dependence of the DM annihilation cross section as expected from the discussion above. Note that $\Gamma_{V}$ depends sensitively on the value of $r=m_{\phi} / m_{V}$ since for $r<1 / 2$, the DP can decay dominantly into DM pairs with a reasonable large partial width, $\Gamma_{V} / m_{V}=g_{D}^{2} \beta^{3} / 48 \pi,{ }^{5}$ where $\beta^{2}=1-4 r^{2}$. However, for larger values of $r$, in the kinematic region of interest to us below, only the DP decay to $\mathrm{SM}$ states is allowed and these modes all have partial widths that are $\epsilon^{2}$ suppressed, e.g., $\Gamma\left(V \rightarrow e^{+} e^{-}\right) / m_{V}=(\epsilon e)^{2} / 12 \pi \lesssim 10^{-(8-10)}$, where the electron mass has again been neglected.

To obtain the thermally averaged cross section leading to the DM relic density we must integrate the above expression weighted by the Bose-Einstein distributions of the incoming complex DM states. Though this is $p$-wave suppressed, the thermal average of the above cross section can yield the observed relic density for the $1 / 2<r<1$ kinematic range of interest as is shown in figure 2. Special care must be taken with the resonance enhancement region $[66,67]$ which we take some minor advantage of in parts of the phase space. For example, if one assumes $g_{D} \epsilon=1 \times 10^{-4}$ and $m_{V}=100 \mathrm{MeV}$, as employed for purposes of demonstration in top panel of figure 2, the required annihilation cross section is obtained near $r \sim 0.55$, within our mass range of interest. Similar other combinations of parameter choices within the mass range $1 / 2<r<1$ can work equally well. As seen in the lower panel of figure 2, fixing $m_{V}=100 \mathrm{MeV}$ for purposed of illustration, we observe that for each value of $r$ there is a unique value of the product $g_{D} \epsilon$ which yields the observed relict density. Then, for any given value of $\epsilon$, the value of $g_{D}$ is completely determined as a function of $r$ and must increase as $r$ increases. This implies that the larger $r$ region will be more sensitive to dark ISR effects since these scale as $g_{D}^{4}$. We will require that value of $g_{D}$ determined in this manner remain perturbative in the analysis below, i.e., $g_{D} \leq \sqrt{4 \pi} \simeq 3.55$; this is relevant as decreases in $\epsilon$ must be compensated for by corresponding increases in the value of $g_{D}$. Since $\sigma_{\mathrm{DM}} v_{\text {rel }} \sim\left(g_{D} \epsilon / m_{V}\right)^{2}$, increasing the value of $m_{V}$ for any fixed value of $r$ will necessitate a similar increase in $g_{D} \epsilon$, apart from the effect of new SM final state mass thresholds, in order to achieve the observed relic density. Away from hadronic resonances the effect of new thresholds is not too large, e.g., for $m_{V}=500 \mathrm{MeV}$, the total annihilation cross section is found to be roughly $\simeq 3.5$ times larger that given by just the $e^{+} e^{-}$mode alone due to the additional contributions arising from muons and pions. Such effects can be easily accounted for in determining the desired values of $g_{D} \epsilon$ to maintain the relic density prediction.

Clearly, we also observe that other equally valid solutions will exist when $r<1 / 2$ but they lie outside the specific mass region of interest to us here. Of course, this overall parameter space will be further constrained by other future experiments, e.g., via dark photon production at accelerators or via DM direct detection experiments employing $\phi$ scattering off of electrons and/or nucleons.

We turn now to our first example of the influence of dark ISR. Consider the specific

\footnotetext{
${ }^{5}$ Here it is assumed that $g_{D}$ is very roughly the size of a typical gauge coupling.
} 

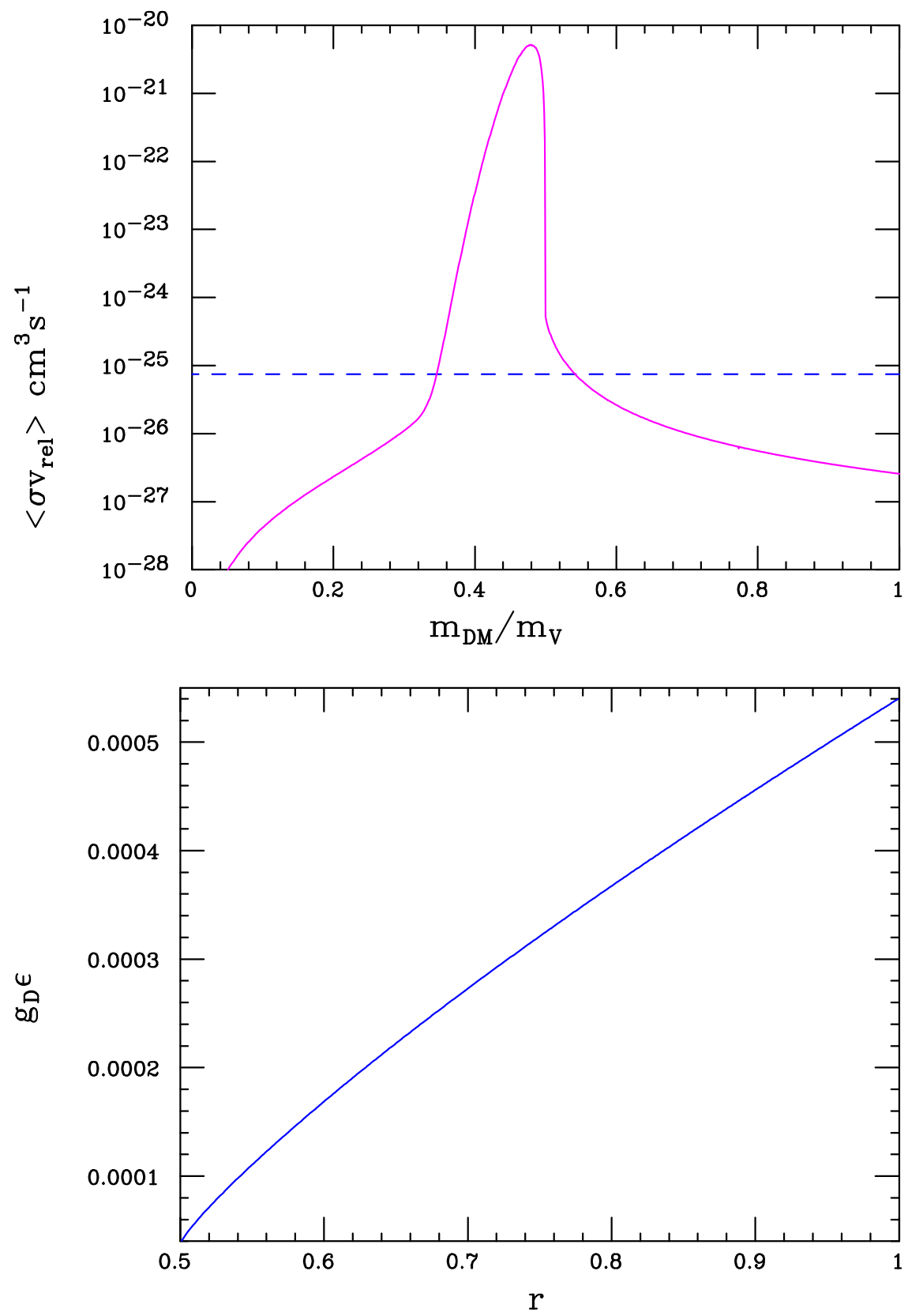

Figure 2. (Top) Sample thermally averaged complex scalar DM annihilation cross section as a function of $r=m_{\phi} / m_{V}$, assuming $m_{V}=100 \mathrm{MeV}$ and $g_{D} \epsilon=10^{-4}$ with $x_{f}=20$. The expression in the square bracket in the text has thus been set to unity here. The corresponding results for other values of these input parameters can be obtained via simple rescaling using the equation in the text. The dashed line shows the approximate annihilation cross section needed to obtain the observed relic density and thus for any $r=m_{\mathrm{DM}} / m_{V}$ in our range of interest the required value of $g_{D} \epsilon$ can be determined. (Bottom) The value of $g_{D} \epsilon$ required to obtain the observed relic density as a function of $r$ in our range of interest assuming that $m_{V}=100 \mathrm{MeV}$ based on the results presented in the top panel. 
process $\phi^{\dagger} \phi \rightarrow V V^{*}, V^{*} \rightarrow e^{+} e^{-}$, which occurs via both $t$ - and $u$-channel DM exchanges as well as via the usual 4-point coupling as noted in figure 1 . We will consider this reaction in the non-relativistic limit for the DM in the mass range of interest $1 / 2<r<1$; we will continue to take $m_{e}=0$ for simplicity in what follows as we will always assume that $m_{\mathrm{DM}} \gg m_{e}$. We note that if $\lambda_{\phi S}$, discussed above, were significant, something that we have assumed not to be the case here, then an additional $s$-channel diagram would be possible via virtual $S^{*}$ exchange followed by $S^{*} \rightarrow V V^{*}$. We find, however, that even when $\lambda_{\phi S}$ is significant, this contribution would be relatively numerically suppressed (by up to one or two orders of magnitude) due to a combination of overall constant factors combined with a relative ratio of $\left(m_{V} / m_{S}\right)^{4} \ll 1$ in comparison to that coming from DP exchange. In any case, we will ignore this possible contribution here.

Recall that these $t-, u$-channel DM exchanges added to the 4-point interaction result in an $s$-wave process and so it is subject to the above mentioned constraints from the CMB. We can write the numerical result for the annihilation cross section for this reaction as

$$
\sigma_{\mathrm{DM}} v_{\mathrm{rel}}=0.216 \sigma_{0} g_{D}^{2}\left(\frac{g_{D} \epsilon}{10^{-4}}\right)^{2}\left(\frac{100 \mathrm{MeV}}{m_{V}}\right)^{2} I,
$$

where $\sigma_{0}=10^{-26} \mathrm{~cm}^{3} \mathrm{~s}^{-1}$ sets the scale to that which is roughly required for the annihilation cross section to result in the observed DM relic density and $I$ is the 3-body 'phase space' integral

$$
I=2 q^{2} \int_{0}^{(1-q)^{2}} d x_{12} \int_{\min }^{\max } d x_{23} \frac{2\left(1-x_{23}\right)\left(x_{12}+x_{23}-q^{2}\right)-x_{12}\left(1+q^{2}+x_{12}\right)}{\left(1-x_{12}-q^{2}\right)^{2}\left[\left(x_{12}-q^{2}\right)^{2}+\left(G q^{2}\right)^{2}\right]},
$$

where $q=1 /(2 r)=m_{V} / 2 m_{\phi}, G=\Gamma_{V} / m_{V}, x_{12}=m_{e e}^{2} / 4 m_{\phi}^{2}$, with $m_{e e}$ being the $e^{+} e^{-}$ invariant mass in the final state, $x_{23}=1-E_{e^{-}} / m_{\phi}$ with $E_{e^{-}}$being the corresponding electron energy, and the range of the $x_{23}$ integration is given by $(\max , \min )=\frac{1}{2}\left(1-x_{12}+\right.$ $\left.q^{2}\right) \pm \frac{1}{2}\left[\left(1-x_{12}-q^{2}\right)^{2}-4 x_{12} q^{2}\right]^{1 / 2}$. In terms of the parameter $q$ appearing in the integral, we note that the $\phi^{\dagger} \phi \rightarrow V^{*} \rightarrow e^{+} e^{-}$resonance region lies in the vicinity of $q \simeq 1$ while the on-shell $\phi^{\dagger} \phi \rightarrow 2 V$ process occurs when $q \sim 1 / 2$. Since the reaction with ISR is an $s$-wave process, the thermal average cross section (sufficiently far from the resonance) is essentially the same as the non-relativistic cross section itself, i.e., $<\sigma_{\mathrm{DM}} v_{\text {rel }}>\simeq \sigma_{\mathrm{DM}} v_{\text {rel }}$, so that numerical results can be obtained in a rather straightforward manner employing the leading term in the familiar velocity expansion. This will be a very good approximation at the time of the $\mathrm{CMB}$ where the DM is quite non-relativistic. Since the DM kinetic decoupling temperature is $\sim 10^{-3} m_{\mathrm{DM}}[46]$, at CMB times the DM temperature, $T_{\mathrm{DM}}(\mathrm{CMB})$, can be roughly estimated as

$$
T_{\mathrm{DM}}(\mathrm{CMB}) \lesssim 10^{-3} m_{\mathrm{DM}}\left(\frac{T_{\mathrm{CMB}}}{10^{-3} m_{\mathrm{DM}}}\right)^{2} \ll m_{\mathrm{DM}},
$$

Thus at the time of the CMB in which we are interested the higher order terms in $v_{\text {rel }}^{2} \sim$ $T_{\mathrm{DM}}(\mathrm{CMB})$ will necessarily be quite highly suppressed and totally ignorable

Our approach will now be as follows: taking $m_{V}=100 \mathrm{MeV}$ for purposes of demonstration, we will input into the above cross section expression the values of $g_{D} \epsilon$ that are required to obtain the observed relic density as a function of $r$ as displayed above in the 

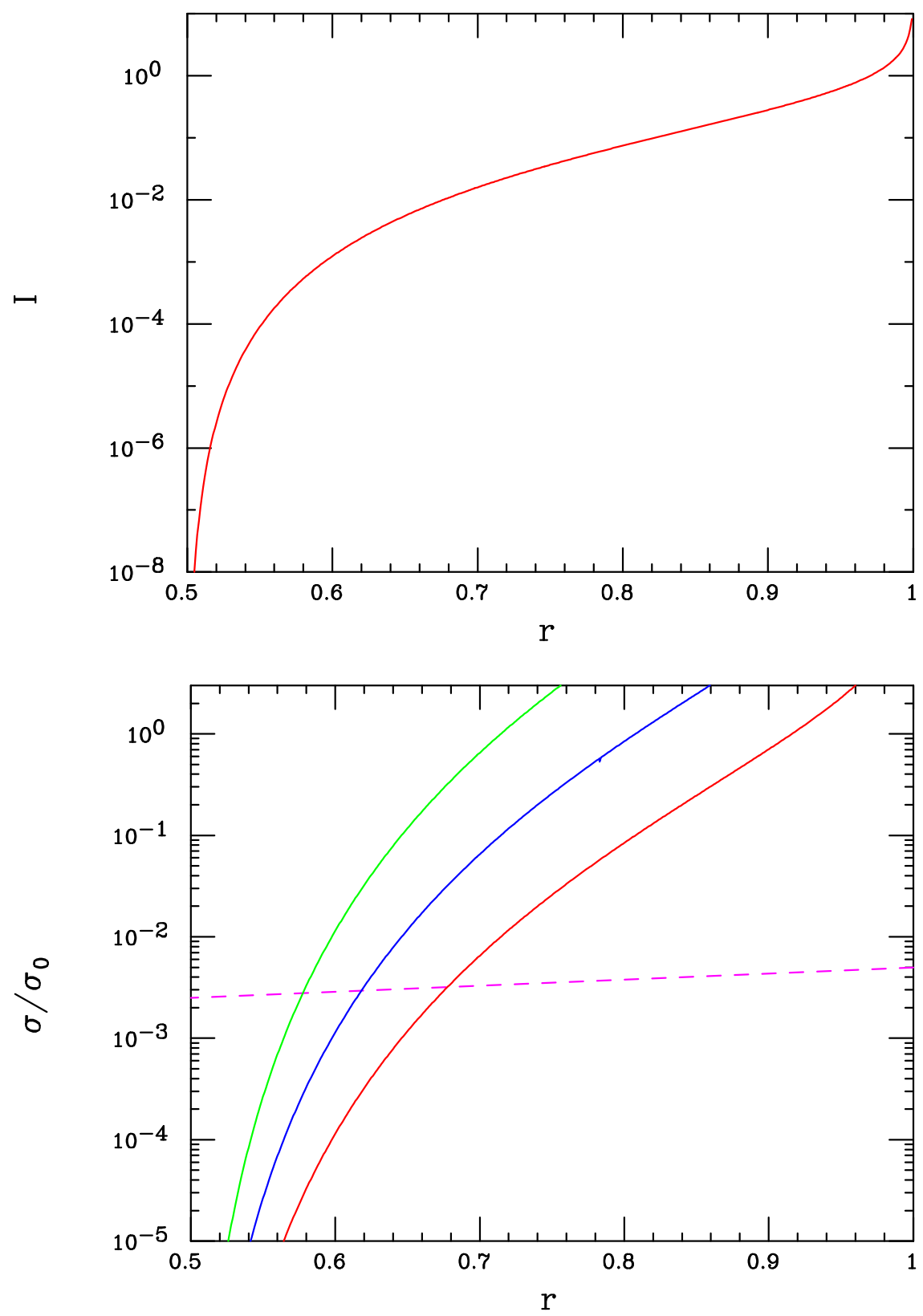

Figure 3. (Top) Value of the phase space integral $I$ as defined in the text as a function of the parameter $r$. Recall that as $r \rightarrow 1$ both DPs can become on-shell. (Bottom) The cross section for the $s$-channel process $\phi^{\dagger} \phi \rightarrow V V^{*}, V^{*} \rightarrow e^{+} e^{-}$with $v_{\text {rel }}^{2}=0$, in units of $\sigma_{0}$, as a function of $r$ assuming, from bottom to top, that $\epsilon=10^{-(3,3.5,4)}$ and assuming that $m_{V}=100 \mathrm{MeV}$. The approximate upper bound on this cross section arising from the CMB [47] is shown as the dashed line. 
lower panel of figure 2. Next, we will assume as further input three representative, phenomenologically interesting values for $\epsilon=10^{-3}, 10^{-3.5}$ and $10^{-4}$, respectively, from which $g_{D}$ can then be obtained as a function of $r$, subject to the perturbativity constraint mentioned above, i.e., $g_{D} \leq \sqrt{4 \pi} \simeq 3.55$. We note that in some cases, that if $r$ is sufficiently large this bound can be violated so some care is required. Note that with the relic density constraint used as input the dark ISR cross section has a practical scaling $\sim \epsilon^{-2}$ which will make the $\mathrm{c}$ ross section larger and hence the CMB bound stronger as $\epsilon$ decreases. Finally, as is well known, the 3-body phase space integral $I$ will itself have a rather strong dependence on the value of $r$ as is shown in the upper panel of figure 3. Combining these factors we then obtain the resulting cross section for the $\phi^{\dagger} \phi \rightarrow V V^{*}, V^{*} \rightarrow e^{+} e^{-}$process as a function of $r$ as shown in the lower panel of figure 3 where we also compare it to the approximate upper limit obtained from the CMB in ref. [47]. Note that along each curve of constant $\epsilon, g_{D}$ is increasing from left to right as $r$ increases to maintain the appropriate DM relic density and its value can be extracted directly from the lower panel of figure 2 .

Several things can be seen from this figure: $(i)$ As we might have expected, as $\epsilon$ decreases the value of $g_{D}$ increases for fixed $m_{V}$ to maintain the correct relic density and this results in an increase in the 3-body cross section for a given value or $r$. (ii) In particular, for $\epsilon=10^{-(3,3.5,4)}$, to satisfy the approximate CMB bound we must have $r \lesssim(0.68,0.62,0.58)$, respectively; larger values of $r$ in each case are thus excluded. These are respectably strong constraints on the model parameter space and as $\epsilon$ becomes smaller we are pushed ever closer to the DP resonance region for DM annihilation to maintain smaller values of $g_{D}$. As noted earlier, since the 3-body cross section effectively scales as $\sim \epsilon^{-2}$ when the relic density is used as a constraint the results for other values of this parameter are easily obtained. (iii) This $r$ bound then results in the further constraint that $g_{D} \lesssim(0.28,0.68,1.67)$, respectively, for these chosen values of $\epsilon$, all of which lie within the perturbatively allowed range. (iv) If the value of $m_{V}$ increases for fixed values of $r$ and $\epsilon$, then $g_{D}$ must also increase to maintain agreement with the observed relic density. This implies that the cross section for the 3-body $\phi^{\dagger} \phi \rightarrow V V^{*}, V^{*} \rightarrow e^{+} e^{-}$process must also grow but now even more rapidly since it scales as $g_{D}^{4}$. For example, a doubling of $m_{V}$ to $200 \mathrm{MeV}$ for fixed values of $(r, \epsilon)$, quadruples the 3-body annihilation rate. However, at the same time, the approximate $\mathrm{CMB}$ cross section constraint is also weakened, i.e., increased by a factor of two so that the ratio of the predicted 3-body cross dark ISR section to the CMB bound only doubles. Still, due to the steep rise of the cross section with increasing $r$, the resulting bound on $r$ strengthens only slightly by roughly $\simeq 0.02$. Further increases in the value of $m_{V}$ will thus only somewhat tighten the constraint on the value of $r$ obtained above. We note that future constraints on this 3 -body process from the CMB are only expected to strengthen by roughly at most a factor of $\sim 2-3$ in the coming few years [48-50] so that given the rapid growth of the 3-body cross section with $r$ the resulting improved restrictions on the parameter space of this model will be rather minor but perhaps not ignorable in a detailed numerical analysis. A final thing that we should notice in the figure is that for large values of $r$ the 3 -body $\phi^{\dagger} \phi \rightarrow V V^{*}, V^{*} \rightarrow e^{+} e^{-}$process could have made a genuine contribution to the DM relic density if this region had not been disallowed by the CMB data. 
It is clear from this analysis that the CMB can place significant constraints on the complex scalar DM model parameter space within the region of interest explicitly explored here. If the DP mass is larger than $100 \mathrm{MeV}$ and/or $\epsilon$ is constrained to be smaller by other experiments, then these constraints will only become stronger.

\section{Pseudo-Dirac fermion DM scenario}

We now turn to the case of a pseudo-Dirac DM particle; here the complex scalar part of $L_{\mathrm{DM}}$ above is replaced by

$$
L_{\mathrm{DM}}=i \bar{\chi} \gamma^{\mu} D_{\mu} \chi-m_{D} \bar{\chi} \chi-\left(y_{s} \bar{\chi} \chi^{c} S+\text { h.c. }\right),
$$

where, in addition to the $\mathrm{U}(1)_{D}$-invariant Dirac mass, $m_{D}$, we assume that there also exists a Majorana mass term, $m_{M}$, which is generated by $\chi$ 's coupling to $S$ after it obtains the dark Higgs vev, $v_{s}$. For this to happen, of course, we need to require that $2 Q_{D}(\chi)+Q_{D}(S)=0$ which is easily arranged. In such a case, the Dirac field $\chi$ splits into two distinct mass eigenstates: $\chi=\left(\chi_{1}+i \chi_{2}\right) / \sqrt{2} i$ and $\chi^{c}=i\left(\chi_{1}-i \chi_{2}\right) / \sqrt{2}$, with $m_{1,2}=m_{D} \mp m_{M}$ where $m_{M}=y_{s} v_{s} / \sqrt{2}$ and $y_{s}$ being a (assumed real) Yukawa coupling. ${ }^{6}$ The fractional mass splitting between these two states is then simply $\delta=\left(m_{2}-m_{1}\right) / m_{1}=2 m_{M} / m_{1}$ which may, in principle, be $O(1)$ or even larger. For values of $\delta \gtrsim 0.01-0.05$ or so, it is unlikely that any signal would be obtained in this scenario from direct detection experiments [68] at tree level since the DM trapped in the galaxy would have insufficient velocity to excite the higher mass state. Here $\chi_{1}$ is identified with the stable DM whereas $\chi_{2}$ can now decay to $\chi_{1}$ plus a (possibly on-shell) DP due to an off-diagonal interaction. For smaller values of $\delta$, where $V$ is clearly off-shell as in the cases we consider here, this lifetime can be fairly long since the decay width roughly scales as $\sim\left(g_{D} e \epsilon\right)^{2} \delta^{5}[69,70]$. In the mass eigenstate basis, this leading order off-diagonal interaction of $\chi_{1,2}$ with the DP, $V$, is given by

$$
L_{\text {int }}=i g_{D}\left(\bar{\chi}_{1} \gamma_{\mu} \chi_{2}-\bar{\chi}_{2} \gamma_{\mu} \chi_{1}\right) V^{\mu}
$$

from which we see immediately why co-annihilation via virtual $s$-channel $V$-exchange is necessary to obtain the observed DM relic density since the 'direct' reactions $\bar{\chi}_{1(2)} \chi_{1(2)} \rightarrow$ $V^{*} \rightarrow e^{+} e^{-}$do not occur at lowest order in $g_{D}$.

As is well-known [71], the co-annihilation process $s$-channel cross section for $\bar{\chi}_{1} \chi_{2}+$ h.c. $\rightarrow V^{*} \rightarrow e^{+} e^{-}$at freeze-out is suppressed by a factor of $\lambda=2 F /(1+F)^{2}$ [71], where $F=(1+\delta)^{3 / 2} e^{-\delta x_{f}}$, in comparison to the naive calculation due to the thermally suppressed $\chi_{2}$ distribution. For $\delta=0.1(0.2,0.3)$ one finds that $F \simeq 0.16(0.024,0.0037)$, assuming that $x_{f}=20$, and falls quite rapidly as $\delta$ increases further - hence the reason why small values of $\delta$ are clearly preferred in obtaining sufficiently large cross sections. In the non-relativistic limit for this $s$-wave process we find that this annihilation cross section, using the notation above (but now with $r=m_{1} / m_{V}$ ), is given by

$$
\sigma_{\mathrm{DM}} v_{\mathrm{rel}}=34.1 \lambda \sigma_{0}\left(\frac{g_{D} \epsilon}{10^{-4}}\right)^{2}\left(\frac{100 \mathrm{MeV}}{m_{V}}\right)^{2} \frac{r^{2}(1+\delta)}{\left[r^{2}(2+\delta)^{2}-1\right]^{2}+G^{2}} .
$$

\footnotetext{
${ }^{6}$ Note that in our notation $\chi_{1}$ is the lighter state and we will for simplicity assume that $m_{M}<m_{D}$ here.
} 
Here we have assumed that the contributions to $\left\langle\sigma_{\mathrm{DM}} v_{\mathrm{rel}}\right\rangle$ from both the $\bar{\chi}_{1} \chi_{1}$ and $\bar{\chi}_{2} \chi_{2}$ channels can be neglected since they appear only at higher order in $g_{D}^{2}$ (as will be discussed below).

At later times, e.g., during the CMB (at roughly $z \sim 10^{3}$ ), this co-annihilation cross section is suppressed by a factor of order $\sim e^{-\delta x_{f}\left(T_{F O} / T_{C M B}\right)}$ as previously noted so that the CMB constraints are simultaneously very easily satisfied. We note that if $m_{1}>m_{V}$ then the $s$-wave cross section for $\bar{\chi}_{1} \chi_{1} \rightarrow 2 V$ with $V$ on-shell is no longer $\epsilon^{2}$ suppressed and can easily lead to conflict with CMB constraints as was the case for $\phi^{\dagger} \phi \rightarrow 2 V$ above; thus we will maintained the requirement that $r=m_{1} / m_{V}<1$ in our calculations here to avoid this issue.

To get a feel for these cross sections we will assume as above that $m_{V}=100 \mathrm{MeV}$ and $g_{D} \epsilon=10^{-4}$ and obtain results as a function of $r$ for various values of the mass splitting $\delta$; for other input parameter choices a simple rescaling employing the equations above is straightforward, once annihilations to additional possible SM final states are appropriately accounted for in the cross section as in the case of complex scalar DM. The calculation is performed as in the complex scalar DM model except that the initial state distributions are now Fermi-Dirac. The output of this calculation is seen in the top panel of figure 4 . The falling behavior of the cross section with increasing $r$ is similar to that obtained in the case of complex scalar DM, but we also see that the cross section declines significantly as expected as the fractional mass splitting, $\delta$, is also increased for fixed $r$. One might expect that as the value of either of these parameters is increased, the value of $g_{D} \epsilon$ required to obtain the observed DM relic density must correspondingly increase and this is indeed the case as shown in the lower panel of this same figure. Thus we expect that the constraints arising from comparisons of dark ISR cross sections with the CMB bounds to be strongest for larger values of either $r$ or $\delta$. Here we also see that the relevant required range of values for the product $g_{D} \epsilon$ is somewhat greater than in the case of complex scalar DM; this is particularly so when $\delta=0.25$ is assumed. As in the complex scalar case, for any assumed value of $\epsilon$ this result also tells us the required value of $g_{D}$. It is to be noted that for smaller values of $\delta$ and fixed values of $r$, these annihilation cross sections are larger than those for complex scalar DM so that it is likely that the resulting constraints from the CMB may also be slightly weaker for small values of $\delta$ than in that case.

With DP ISR, the $s$-wave process $\bar{\chi}_{1} \chi_{1} \rightarrow V V^{*}, V^{*} \rightarrow e^{+} e^{-}$becomes possible when $1 / 2<r<1$ where the $\chi_{2}$ is now exchanged in the $t-$ and $u$-channels as in figure 1 ; the corresponding process with $\chi_{1}$ interchanged with $\chi_{2}$ still remains doubly Boltzmann suppressed. In this case one may worry that the Majorana mass term above now allows for a potentially large coupling $\sim m_{M} / v_{s}$ of $\bar{\chi}_{1} \chi_{1}$ to $S$ so that $S$-exchange itself could directly mediate the $\bar{\chi}_{1} \chi_{1} \rightarrow V V^{*}$ process. While true, it is easy to convince oneself that this process is necessarily dominantly $p$-wave and so that is suppressed by $v_{\text {rel }}^{2} \sim T$ at the later, CMB times of relevance here.

Again employing the non-relativistic limit, we can obtain the $\bar{\chi}_{1} \chi_{1} \rightarrow V V^{*}, V^{*} \rightarrow e^{+} e^{-}$ annihilation rate which can be written in a manner identical to that obtained for the 

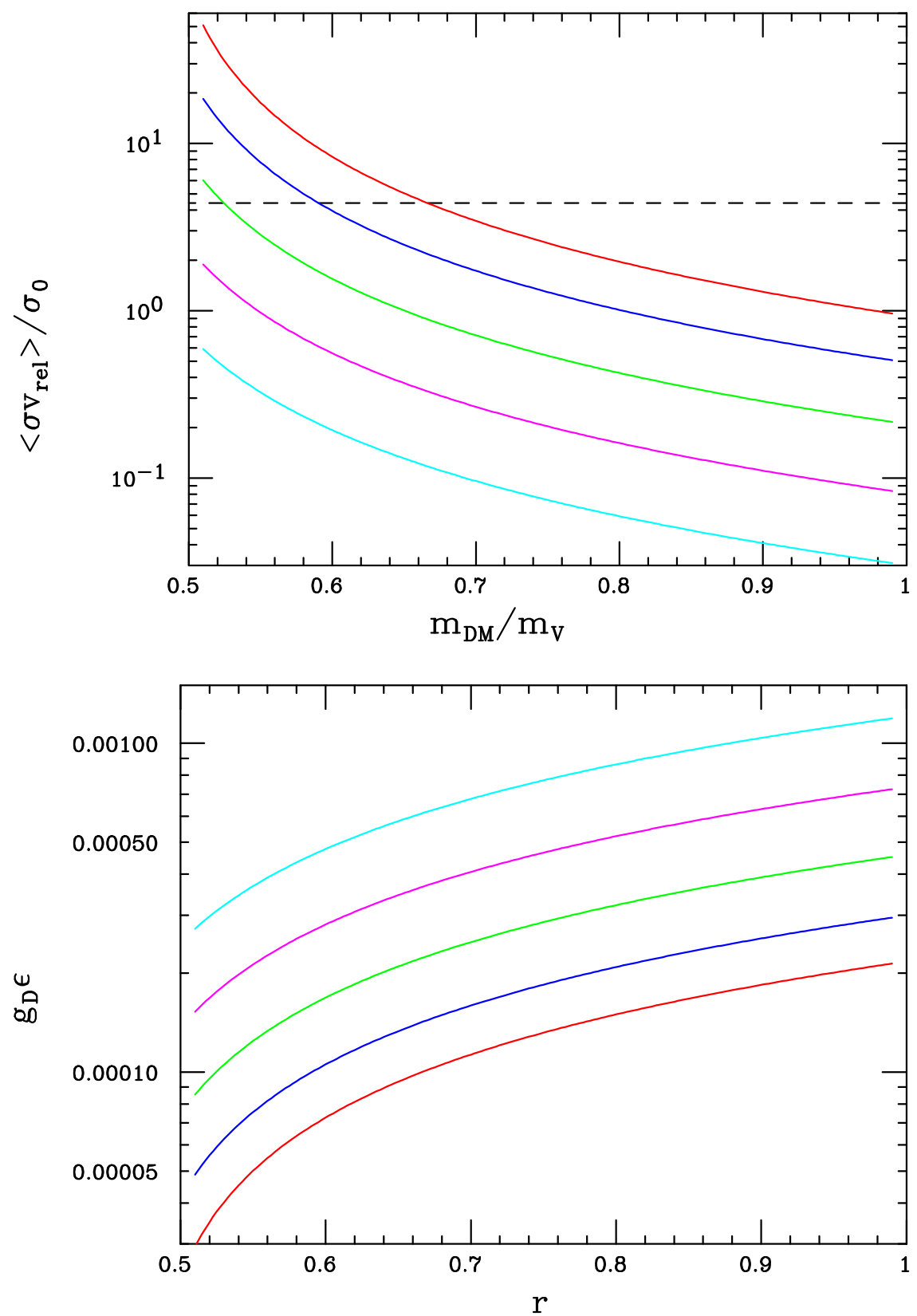

Figure 4. (Top) DM co-annihilation cross section in units of $\sigma_{0}$ assuming that $g_{D} \epsilon=10^{-4}$ and $m_{V}=100 \mathrm{MeV}$ for purposes of demonstration as a function of $r=m_{\mathrm{DM}} / m_{V}$. From top to bottom the curves correspond to the choices of $\delta=0.05,0.10,0.15,0.20$ and 0.25 , respectively. The dashed line represents the approximate cross section needed to obtain the observed relic density. Results for other values of the parameters can be obtained by simple rescaling using the results in the text. (Bottom) Values of $g_{D} \epsilon$ required to obtain the observed relic density employing the same parameter values with curves labelled in reverse order from the top panel. 


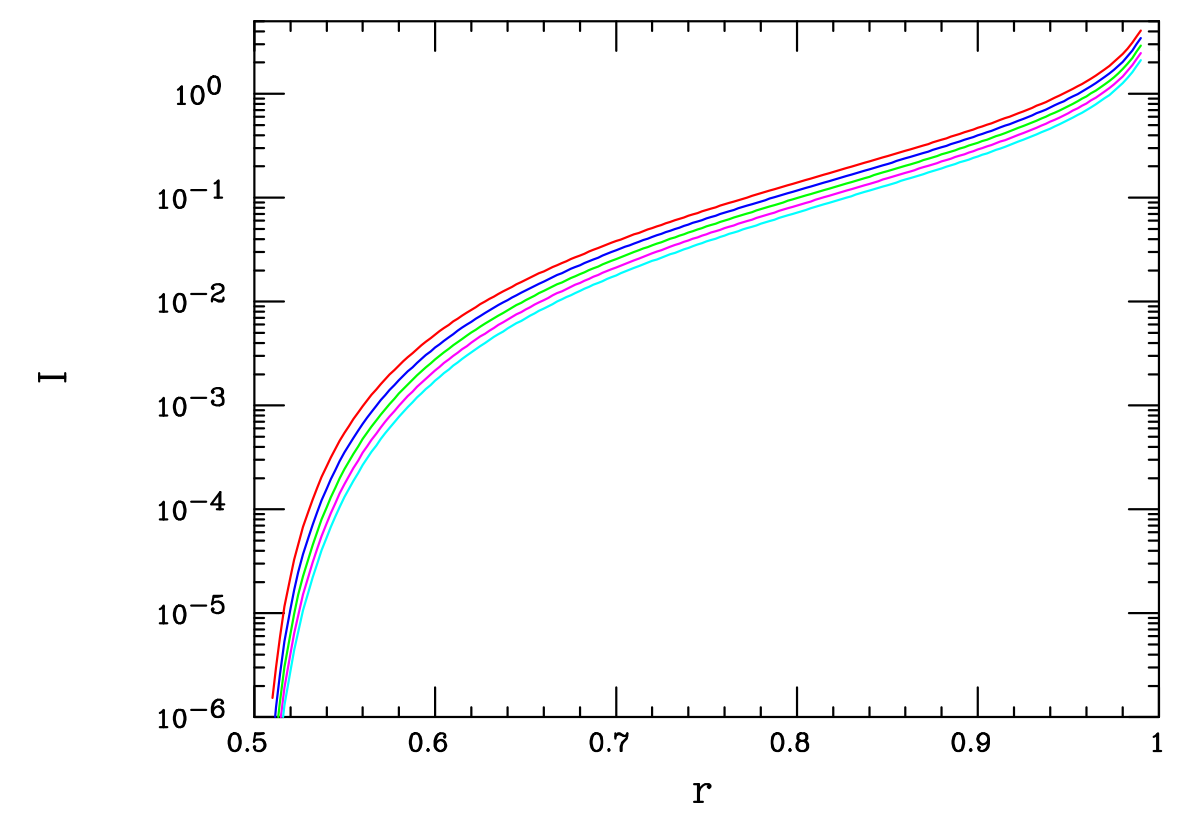

Figure 5. The phase space integral $I$ as defined in the text as a function of $r$ for the case of psuedoDirac DM; from top to bottom the curves correspond to the choices of $\delta=0.05,0.10,0.15,0.20$ and 0.25 , respectively, as discussed in the text.

complex scalar DM case above and is given by

$$
\sigma_{\mathrm{DM}} v_{\mathrm{rel}}=0.216 \sigma_{0} g_{D}^{2}\left(\frac{g_{D} \epsilon}{10^{-4}}\right)^{2}\left(\frac{100 \mathrm{MeV}}{m_{V}}\right)^{2} I,
$$

where, employing the abbreviation $q=1 /(2 r)=m_{V} / 2 m_{1}$ as above, the phase space integral is just $I=2 q^{2} J$ with $J$ being explicitly given by

$$
J=\int_{0}^{(1-q)^{2}} d x_{12} \int_{\min }^{\max } d x_{23} \frac{\left(1-x_{12}+q^{2}\right)\left[\left(1-x_{13}\right)\left(x_{13}-q^{2}\right)+(1 \rightarrow 2)\right]-\left(x_{13}-q^{2}\right)\left(x_{23}-q^{2}\right)\left(1+q^{2}\right)}{\left[\left(x_{12}-q^{2}\right)^{2}+\left(G q^{2}\right)^{2}\right]\left[1-x_{12}-q^{2}+\left(z^{2}-1\right) / 2\right]^{2}},
$$

where we follow the same notation as above and also define the quantities $z=m_{2} / m_{1}=$ $1+\delta$ and $x_{13}=1+q^{2}-x_{12}-x_{23}=1-E_{e^{+}} / m_{1}$. Figure 5 shows the numerical results for this phase space integral $I$ as a function of $r$ for various assumed values of $\delta$. As in the case of complex scalar DM, $I$ shows a very strong $r$-dependence but whose overall behavior and shape is not much influenced by the different assumed values of $\delta$ in the range we have examined. Of course, as $\delta$ increases for fixed $r, I$ does indeed decrease by up to factors of a few, but for the range of values shown this effect is rather slight in comparison to the strong observed $r$-dependence that we obtain here.

We now proceed as we did earlier in the case of complex scalar DM. We assume that $m_{V}=100 \mathrm{MeV}$ for purposes of demonstration and then input the values of $g_{D} \epsilon$ that are required to obtain the observed relic density as a function of $r$ as found above for different assumed values of $\delta$. We then assume as input the same three representative values for $\epsilon=10^{-3}, 10^{-3.5}$ and $10^{-4}$, respectively, as we did before from which $g_{D}$ can then be obtained as a function of both $r$ and $\delta$. As in the case of complex scalar DM we will 

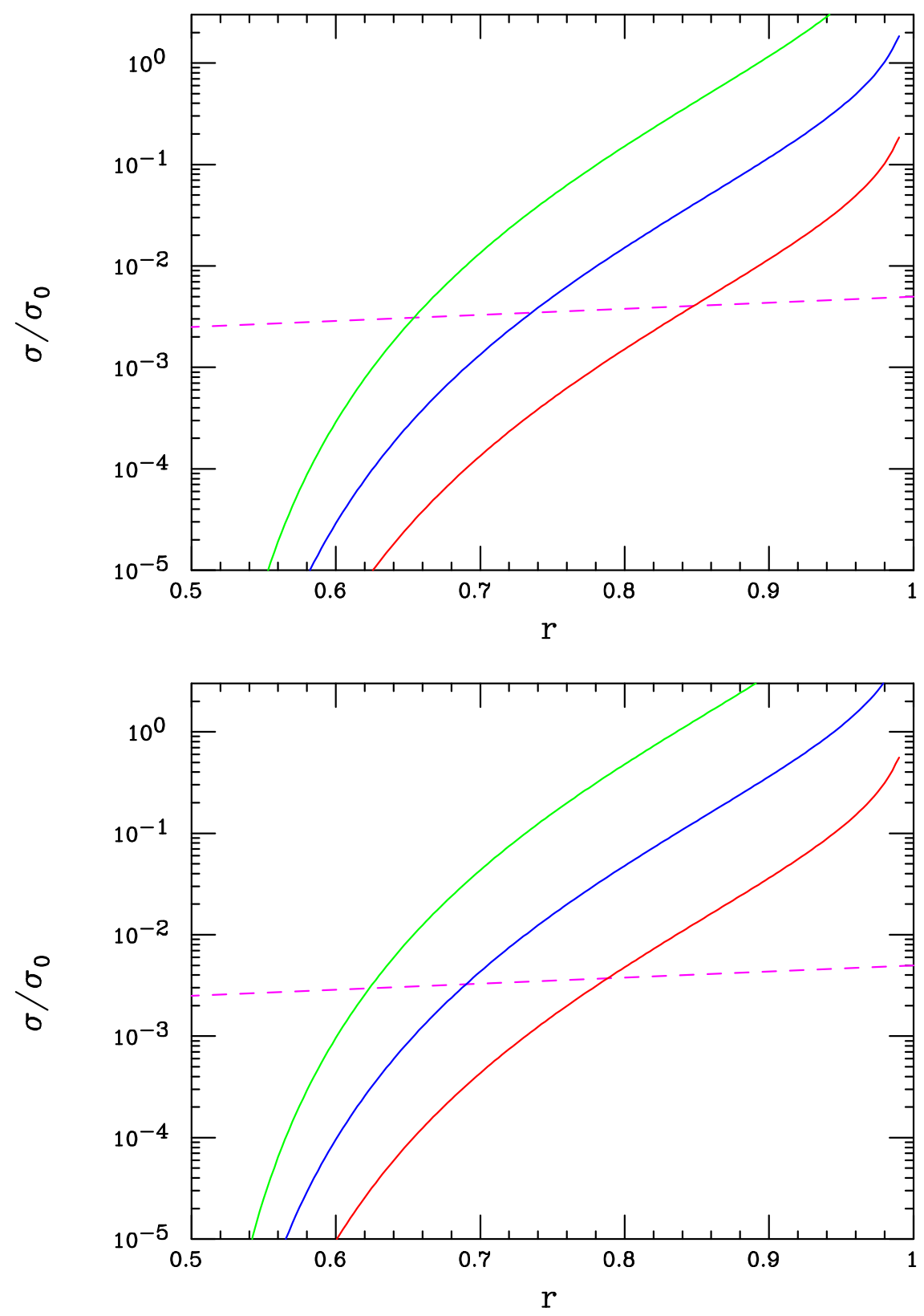

Figure 6. (Top) $s$-wave 3-body DP ISR annihilation cross section as a function of $r$ in units of $\sigma_{0}$ assuming $\delta=0.05$ and $m_{V}=100 \mathrm{MeV}$ with, from bottom to top, $\epsilon=10^{-(3,3.5,4)}$. The approximate upper limit on this cross section arising from the CMB [47] is also shown as the dashed line. (Bottom) Same as the top panel but now with $\delta=0.1$.

always subject the value of $g_{D}$ to the perturbativity constraint as employed above, i.e., $g_{D} \leq \sqrt{4 \pi} \simeq 3.55$. As in the case of complex scalar DM, we note that along each curve of constant $\epsilon, \delta$, the value of $g_{D}$ will increase from left to right along the curve as $r$ increases to maintain the value of the observed relic density and whose value can be extracted from the lower panel of figure 4. 
The results of this calculation for the assumed range values of $\delta=0.05-0.25$ can be found in the set of figures 6,7 and 8 which show the cross section for the process $\bar{\chi}_{1} \chi_{1} \rightarrow$ $V V^{*}, V^{*} \rightarrow e^{+} e^{-}$with $v_{\text {rel }}^{2}=0$ in units of $\sigma_{0}$ as a function of $r$ for the three values of $\epsilon$ chosen above taking $m_{V}=100 \mathrm{MeV}$. Also shown is the approximate constraint arising from the CMB [47]. In all 5 of these cases the overall qualitative nature of the results are similar to what was found for the case of complex scalar DM but the details are observed to be quite different. For fixed values of $\delta$ and $\epsilon$ the cross section rises quickly with increasing $r$ due to both the rapid opening of the phase space as well as the increasing value of $g_{D}$ as previously observed. As an example, for a fixed value of $\delta=0.05[0.1]$, assuming that $\epsilon=10^{-3}, 10^{-3.5}$ and $10^{-4}$ leads to the constraint $r \lesssim 0.85,0.74,0.65[0.79,069.0 .62]$, respectively. However, as $\delta$ increases for fixed $\epsilon$ we also see that the bound arising from the CMB rapidly becomes stronger as might be expected since for fixed values of $r$ and $\epsilon$ the value of $g_{D}$ must increase to obtain the observed relic density. For example, assuming that $\epsilon=10^{-3.5}$ we obtain an upper limit of $r \lesssim 0.74(0.69,0.64,0.60,0.57)$ for $\delta=0.05(0.1,0.15,0.2,0.25)$, respectively. One difference we see with the earlier result for the complex scalar case is that when $\delta=0.2,0.25$ and $\epsilon=10^{-4}$ the predicted cross section curve terminates in the middle of the plot but still within the now excluded region. This is the result of imposing the perturbativity constraint on $g_{D}$ as discussed above, i.e., $g_{D} \leq \sqrt{4 \pi} \simeq 3.55$, i.e., larger values of $r$ in these case requires values of $g_{D}$ exceeding the perturbativity bound in order to reach the desired relic density. These values of $g_{D}$ can be read off directly by using the lower panel of figure 4 for any given value of $\epsilon$. As in the scalar DM case above, we again should notice in the figures is that for larger values of $r$ the 3-body process could have made a genuine contribution to the DM relic density if this region had not already been disallowed by the CMB data.

As was observed in the previously examined case of complex scalar DM, it is clear from this analysis that the CMB can place significant constraints on the pseudo-Dirac DM model parameter space. If the DP mass is larger than $100 \mathrm{MeV}, \epsilon$ is constrained to take on values smaller than those considered here by other experiments, or the mass splitting between the two fermion states is for some reason larger, then in any of these cases the constraints shown above will only become stronger. Clearly this is an important constraint on this model in this kinematic range.

\section{Discussion and summary}

The kinetic mixing model with both dark photons and dark matter in the sub-GeV mass range poses an interesting alternative to the well-studied traditional WIMP and axion scenarios. However, constraints from the CMB can pose significant model building requirements on this class of models in that the annihilation process by which the DM achieves the observed relic density cannot be dominantly $s$-wave, or more generally, temperatureindependent. Annihilation via a $p$-wave process, as in the case of complex scalar DM, or via co-annihilation, as in the case of pseudo-Dirac DM with a small mass splitting, offer two attractive alternative setups that circumvent these constraints. In both these scenarios, the emission of additional dark ISR in the form of a DP as part of the annihilation 

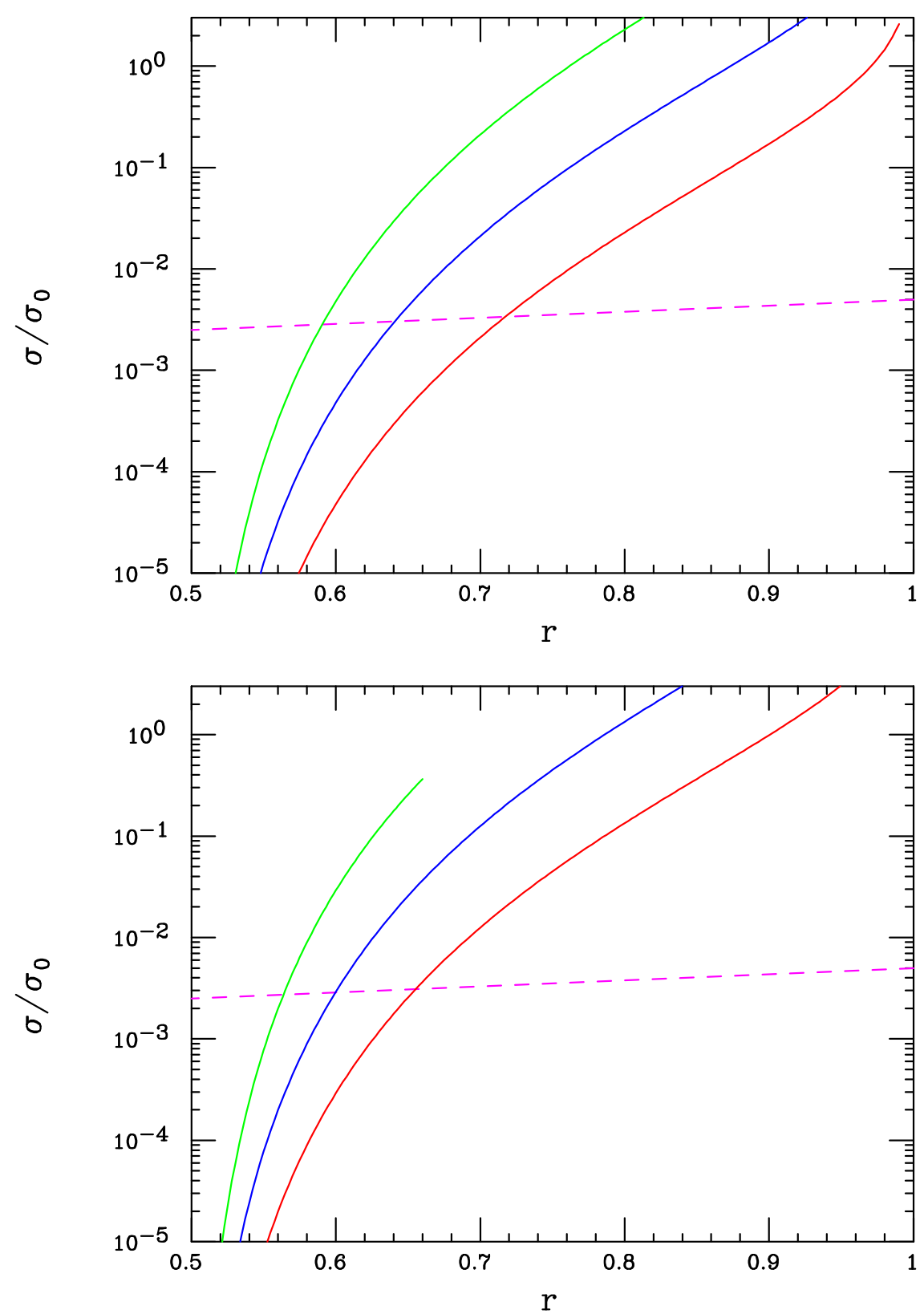

Figure 7. Same as the previous figure but now assuming that $\delta=0.15$ (Top) or $\delta=0.2$ (Bottom). Note the termination of the curve for $\epsilon=10^{-4}$ in the middle of the plot due to the perturbativity constraint on $g_{D}$. 


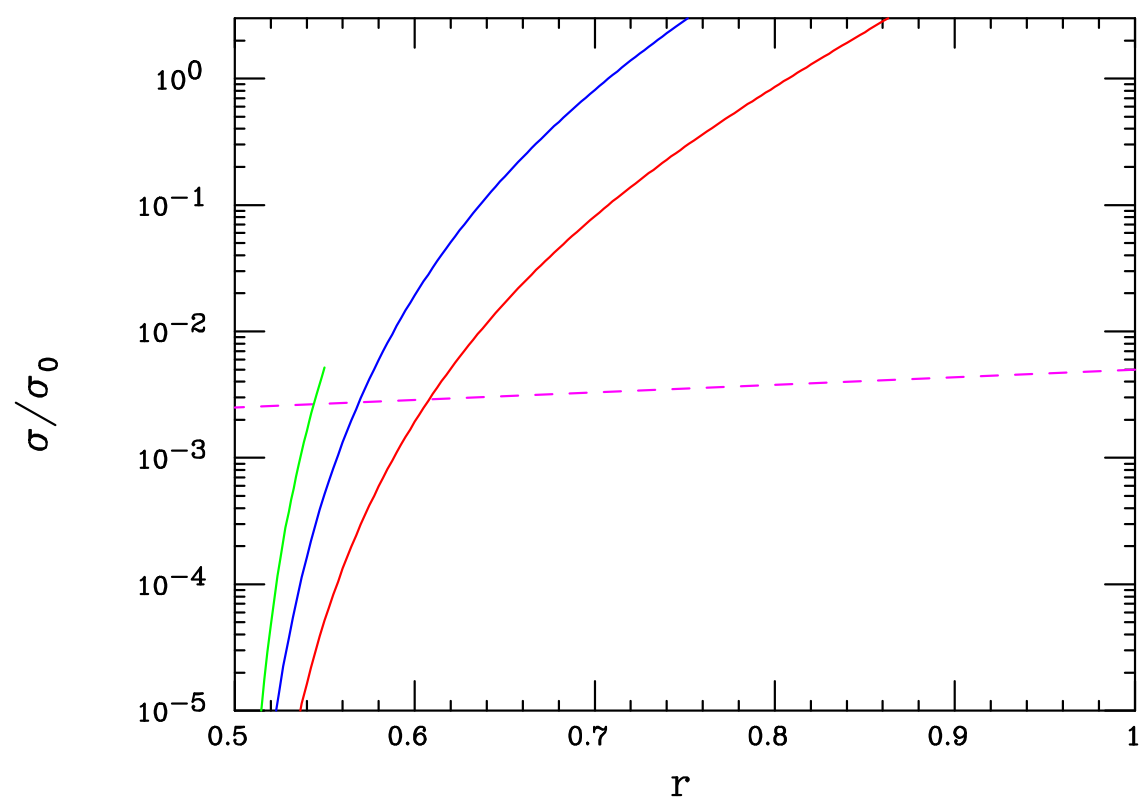

Figure 8. Same as the previous figure but now assuming that $\delta=0.25$. Again the perturbativity constraint on $g_{D}$ is playing a role here when $\epsilon=10^{-4}$.

process, though higher order in $g_{D}$ and a 3-body process, necessarily leads to a numerically suppressed $s$-wave process with a rate that is insufficient to explain the DM relic density but may still be in conflict with the bounds from the CMB in certain parameter space regimes. This leads to an additional set of constraints on the model space of these scenarios and, in this paper, we performed a preliminary examination of the impact of these constraints arising from the rate for the emission of this additional DP. Indeed, potentially important constraints were obtained on the parameter spaces for both the complex scalar and pseudo-Dirac scenarios once the necessity of achieving the observed DM relic density was simultaneously imposed.

For both of these DM models we followed the same procedure: fixing the DP mass to $100 \mathrm{MeV}$, we first determined the value of the product $g_{D} \epsilon$ as a function on the DM to DP mass ratio, $r$, required to obtain the observed DM relic density. In the pseudo-Dirac case this analysis was performed for several values of the mass splitting between the two states, $\delta$. Using scaling and the measured value of $R\left(e^{+} e^{-} \rightarrow\right.$ hadrons $)$, the corresponding result can be obtained for any value of $m_{V}$; larger values of $m_{V}$ generally require larger values of $g_{D} \epsilon$ for fixed $r$. These results were then employed as input into the dark ISR rate calculation for several fixed values of $\epsilon$ and results for any other set of values of $\epsilon$ are easily obtainable from simple scaling. This fixed the numerical value of $g_{D}$ and only those parameter space points where $g_{D}$ remained perturbative, i.e., $g_{D} \leq \sqrt{4 \pi} \simeq 3.55$, were kept.

For both the complex scalar and pseudo-Dirac DM models with the DP and DM within the ranges considered here, rather strong constraints on the relevant parameter spaces were obtained. Though qualitatively similar, these constraints differ in detail between these two types of models. In both models, for fixed values of the DP mass, $m_{V}$, as well as for fixed $\delta$ in the pseudo-Dirac DM case, when the KM parameter, $\epsilon$ becomes smaller, 
a correspondingly larger value of the dark gauge coupling, $g_{D}$, is needed to obtain the necessary DM annihilation cross section for a fixed value of the ratio of the DM and DP masses, $1 / 2<r=m_{\mathrm{DM}} / m_{\mathrm{DP}}<1$. As this mass ratio increases, since the cross section for dark ISR scales as $g_{D}^{4}$, regions of the parameter with larger values of $r$ are found to be more constrained - and indeed become excluded - by the CMB measurements. Since $g_{D}$ must increase when $m_{V}$ increases, taking all other parameters fixed, the bound on $r$ from the CMB only becomes stronger. Similarly, in the pseudo-Dirac DM model, increasing $\delta$ with all other parameters fixed also strengthens the bound on $r$ with part of the parameter space being eliminated by the perturbativity requirement on $g_{D}$. For smaller values of $\delta \lesssim 0.05-0.1$, the bounds obtained on $r$ are somewhat weaker in the pseudo-Dirac case in comparison to the complex scalar DM model due to the somewhat larger cross section for co-annihilation. However, for larger values of $\delta \gtrsim 0.2-0.25$ this situation is reversed since the co-annihilation cross section is now more highly Boltzmann suppressed. Furthermore, in both of these models, decreasing the value of $\epsilon$ itself while holding everything else fixed raises the dark ISR cross section as it scales as $\epsilon^{-2}$. Again, this will lead to even stronger constraints on the other model parameters.

From the above analysis it is clear that further detailed study of the effects of the CMB constraints and dark ISR on the parameter spaces of light DM models is warranted.

\section{Acknowledgments}

The author would like to thank J.L. Hewett and G. Wojcik for valuable discussions related to this analysis, Yu-Dai Tsai for both discussions and various numerical checks, and also D. Rueter for both discussions and for providing the Feynman diagrams used within. The author would also like to thank T. Slatyer for bringing the work in refs. [44, 45] to his attention. This work was supported by the Department of Energy, Contract DE-AC02$76 \mathrm{SF} 00515$.

Open Access. This article is distributed under the terms of the Creative Commons Attribution License (CC-BY 4.0), which permits any use, distribution and reproduction in any medium, provided the original author(s) and source are credited.

\section{References}

[1] G. Arcadi et al., The waning of the WIMP? A review of models, searches, and constraints, Eur. Phys. J. C 78 (2018) 203 [arXiv:1703.07364] [INSPIRE].

[2] L. Roszkowski, E.M. Sessolo and S. Trojanowski, WIMP dark matter candidates and searches-current status and future prospects, Rept. Prog. Phys. 81 (2018) 066201 [arXiv: 1707.06277] [INSPIRE].

[3] M. Kawasaki and K. Nakayama, Axions: theory and cosmological role, Ann. Rev. Nucl. Part. Sci. 63 (2013) 69 [arXiv:1301.1123] [INSPIRE].

[4] P.W. Graham, I.G. Irastorza, S.K. Lamoreaux, A. Lindner and K.A. van Bibber, Experimental searches for the axion and axion-like particles, Ann. Rev. Nucl. Part. Sci. 65 (2015) 485 [arXiv: 1602.00039] [inSPIRE]. 
[5] I.G. Irastorza and J. Redondo, New experimental approaches in the search for axion-like particles, Prog. Part. Nucl. Phys. 102 (2018) 89 [arXiv:1801.08127] [InSPIRE].

[6] K. Pachal, Dark matter searches at ATLAS and CMS, given at the $8^{\text {th }}$ edition of the Large Hadron Collider Physics Conference, May 25-30 (2020).

[7] XENON collaboration, Dark matter search results from a one ton-year exposure of XENON1T, Phys. Rev. Lett. 121 (2018) 111302 [arXiv:1805.12562] [INSPIRE].

[8] Fermi-LAT, DES collaboration, Searching for dark matter annihilation in recently discovered Milky Way satellites with Fermi-LAT, Astrophys. J. 834 (2017) 110 [arXiv: 1611.03184] [INSPIRE].

[9] PICO collaboration, Dark matter search results from the complete exposure of the PICO-60 $C_{3} F_{8}$ bubble chamber, Phys. Rev. D 100 (2019) 022001 [arXiv:1902.04031] [InSPIRE].

[10] J. Alexander et al., Dark sectors 2016 workshop: community report, arXiv:1608.08632 [INSPIRE].

[11] M. Battaglieri et al., US cosmic visions: new ideas in dark matter 2017: community report, arXiv: 1707.04591 [INSPIRE].

[12] G. Bertone and M.P. Tait, Tim, A new era in the search for dark matter, Nature 562 (2018) 51 [arXiv: 1810.01668 ] [INSPIRE].

[13] G. Steigman, CMB Constraints On The Thermal WIMP Mass And Annihilation Cross Section, Phys. Rev. D 91 (2015) 083538 [arXiv:1502.01884] [INSPIRE].

[14] K. Saikawa and S. Shirai, Precise WIMP dark matter abundance and standard model thermodynamics, JCAP 08 (2020) 011 [arXiv: 2005.03544] [INSPIRE].

[15] B. Holdom, Two U(1)'s and epsilon charge shifts, Phys. Lett. B 166 (1986) 196 [InSPIRE].

[16] B. Holdom, Searching for $\epsilon$ charges and a new U(1), Phys. Lett. B 178 (1986) 65 [inSPIRE].

[17] K.R. Dienes, C.F. Kolda and J. March-Russell, Kinetic mixing and the supersymmetric gauge hierarchy, Nucl. Phys. B 492 (1997) 104 [hep-ph/9610479] [INSPIRE].

[18] F. Del Aguila, The physics of $Z^{\prime}$ bosons, Acta Phys. Polon. B 25 (1994) 1317 [hep-ph/9404323] [INSPIRE].

[19] K.S. Babu, C.F. Kolda and J. March-Russell, Leptophobic U(1)s and the R(b)-R(c) crisis, Phys. Rev. D 54 (1996) 4635 [hep-ph/9603212] [InSPIRE].

[20] T.G. Rizzo, Gauge kinetic mixing and leptophobic $Z^{\prime}$ in $E_{6}$ and $\mathrm{SO}(10)$, Phys. Rev. D 59 (1998) 015020 [hep-ph/9806397] [INSPIRE].

[21] D. Feldman, B. Körs and P. Nath, Extra-weakly interacting dark matter, Phys. Rev. D 75 (2007) 023503 [hep-ph/0610133] [INSPIRE].

[22] D. Feldman, Z. Liu and P. Nath, The Stueckelberg $Z^{\prime}$ extension with kinetic mixing and milli-charged dark matter from the hidden sector, Phys. Rev. D 75 (2007) 115001 [hep-ph/0702123] [INSPIRE].

[23] M. Pospelov, A. Ritz and M.B. Voloshin, Secluded WIMP dark matter, Phys. Lett. B 662 (2008) 53 [arXiv:0711.4866] [INSPIRE].

[24] M. Pospelov, Secluded U(1) below the weak scale, Phys. Rev. D 80 (2009) 095002 [arXiv:0811.1030] [INSPIRE]. 
[25] H. Davoudiasl, H.-S. Lee and W.J. Marciano, Muon anomaly and dark parity violation, Phys. Rev. Lett. 109 (2012) 031802 [arXiv: 1205.2709] [INSPIRE].

[26] H. Davoudiasl, H.-S. Lee and W.J. Marciano, 'Dark' Z implications for parity violation, rare meson decays, and Higgs physics, Phys. Rev. D 85 (2012) 115019 [arXiv:1203.2947] [INSPIRE].

[27] R. Essig et al., Working group report: new light weakly coupled particles, arXiv:1311.0029 [INSPIRE].

[28] E. Izaguirre, G. Krnjaic, P. Schuster and N. Toro, Analyzing the discovery potential for light dark matter, Phys. Rev. Lett. 115 (2015) 251301 [arXiv:1505.00011] [INSPIRE].

[29] M. Khlopov, Fundamental particle structure in the cosmological dark matter, Int. J. Mod. Phys. A 28 (2013) 1330042 [arXiv:1311.2468] [InSPIRE].

[30] D. Curtin, R. Essig, S. Gori and J. Shelton, Illuminating dark photons with high-energy colliders, JHEP 02 (2015) 157 [arXiv:1412.0018] [INSPIRE].

[31] M. Fabbrichesi, E. Gabrielli and G. Lanfranchi, The dark photon, arXiv:2005.01515 [INSPIRE].

[32] T.G. Rizzo, Kinetic mixing and portal matter phenomenology, Phys. Rev. D 99 (2019) 115024 [arXiv:1810.07531] [INSPIRE].

[33] T.D. Rueter and T.G. Rizzo, Towards a UV-model of kinetic mixing and portal matter, Phys. Rev. D 101 (2020) 015014 [arXiv: 1909.09160] [INSPIRE].

[34] J.H. Kim, S.D. Lane, H.-S. Lee, I.M. Lewis and M. Sullivan, Searching for dark photons with maverick top partners, Phys. Rev. D 101 (2020) 035041 [arXiv: 1904.05893] [INSPIRE].

[35] T.G. Rizzo and G.N. Wojcik, Kinetic mixing, dark photons and extra dimensions III: brane localized dark matter, arXiv: 2006.06858 [INSPIRE].

[36] R.G. Landim and T.G. Rizzo, Thick branes in extra dimensions and suppressed dark couplings, JHEP 06 (2019) 112 [arXiv:1902.08339] [INSPIRE].

[37] T.G. Rizzo, Kinetic mixing, dark photons and extra dimensions. Part II: fermionic dark matter, JHEP 10 (2018) 069 [arXiv:1805.08150] [INSPIRE].

[38] T.G. Rizzo, Kinetic mixing, dark photons and an extra dimension. Part I, JHEP 07 (2018) 118 [arXiv: 1801.08525$]$ [INSPIRE].

[39] N. Sabti, J. Alvey, M. Escudero, M. Fairbairn and D. Blas, Refined bounds on MeV-scale thermal dark sectors from BBN and the CMB, JCAP 01 (2020) 004 [arXiv:1910.01649] [INSPIRE].

[40] Planck collaboration, Planck 2018 results. VI. Cosmological parameters, Astron. Astrophys. 641 (2020) A6 [arXiv: 1807.06209] [INSPIRE].

[41] T.R. Slatyer, Indirect dark matter signatures in the cosmic dark ages. I. Generalizing the bound on s-wave dark matter annihilation from Planck results, Phys. Rev. D 93 (2016) 023527 [arXiv: 1506.03811] [INSPIRE].

[42] H. Liu, T.R. Slatyer and J. Zavala, Contributions to cosmic reionization from dark matter annihilation and decay, Phys. Rev. D 94 (2016) 063507 [arXiv: 1604.02457] [INSPIRE].

[43] R.K. Leane, T.R. Slatyer, J.F. Beacom and K.C.Y. Ng, GeV-scale thermal WIMPs: Not even slightly ruled out, Phys. Rev. D 98 (2018) 023016 [arXiv:1805.10305] [INSPIRE]. 
[44] M. Boudaud, J. Lavalle and P. Salati, Novel cosmic-ray electron and positron constraints on MeV dark matter particles, Phys. Rev. Lett. 119 (2017) 021103 [arXiv:1612.07698] [INSPIRE].

[45] M. Boudaud, T. Lacroix, M. Stref and J. Lavalle, Robust cosmic-ray constraints on p-wave annihilating MeV dark matter, Phys. Rev. D 99 (2019) 061302 [arXiv:1810.01680] [INSPIRE].

[46] T. Bringmann and S. Hofmann, Thermal decoupling of WIMPs from first principles, JCAP 04 (2007) 016 [Erratum ibid. 03 (2016) E02] [hep-ph/0612238] [INSPIRE].

[47] J. Cang, Y. Gao and Y.-Z. Ma, Probing dark matter with future CMB measurements, Phys. Rev. D 102 (2020) 103005 [arXiv:2002.03380] [INSPIRE].

[48] D. Green, P.D. Meerburg and J. Meyers, Aspects of dark matter annihilation in cosmology, JCAP 04 (2019) 025 [arXiv: 1804.01055] [INSPIRE].

[49] Simons ObSERvatory collaboration, The Simons Observatory: science goals and forecasts, JCAP 02 (2019) 056 [arXiv: 1808.07445] [INSPIRE].

[50] CMB-S4 collaboration, CMB-S4 science book, first edition, arXiv: 1610.02743 [INSPIRE].

[51] N.F. Bell, J.B. Dent, T.D. Jacques and T.J. Weiler, Electroweak Bremsstrahlung in dark matter annihilation, Phys. Rev. D 78 (2008) 083540 [arXiv:0805.3423] [INSPIRE].

[52] M. Kachelriess, P.D. Serpico and M. Solberg, On the role of electroweak bremsstrahlung for indirect dark matter signatures, Phys. Rev. D 80 (2009) 123533 [arXiv:0911.0001] [INSPIRE].

[53] T. Bringmann, X. Huang, A. Ibarra, S. Vogl and C. Weniger, Fermi LAT search for internal Bremsstrahlung signatures from dark matter annihilation, JCAP 07 (2012) 054 [arXiv: 1203.1312] [INSPIRE].

[54] N.F. Bell, Y. Cai, J.B. Dent, R.K. Leane and T.J. Weiler, Enhancing dark matter annihilation rates with dark bremsstrahlung, Phys. Rev. D 96 (2017) 023011 [arXiv: 1705.01105] [INSPIRE].

[55] HPS collaboration, Search for a dark photon in electroproduced $e^{+} e^{-}$pairs with the Heavy Photon Search experiment at JLab, Phys. Rev. D 98 (2018) 091101 [arXiv:1807.11530] [INSPIRE].

[56] LHCb collaboration, Searches for dark photons in LHCb, PoS (LHCP2019) 180 [INSPIRE].

[57] FASER collaboration, FASER: ForwArd Search ExpeRiment at the LHC, arXiv: 1901.04468 [INSPIRE].

[58] NA64 collaboration, Improved limits on a hypothetical $X(16.7)$ boson and a dark photon decaying into $e^{+} e^{-}$pairs, Phys. Rev. D 101 (2020) 071101 [arXiv:1912.11389] [InSPIRE].

[59] MATHUSLA collaboration, Explore the lifetime frontier with MATHUSLA, 2020 JINST 15 C06026 [arXiv: 1901.04040] [INSPIRE].

[60] BELLE II collaboration, Dark sector physics with Belle II, PoS(LeptonPhoton2019)063 [INSPIRE].

[61] SHIP collaboration, SND@LHC, arXiv:2002.08722 [INSPIRE].

[62] LDMX collaboration, Light Dark Matter eXperiment (LDMX), arXiv:1808.05219 [INSPIRE]. 
[63] A. Berlin, N. Blinov, G. Krnjaic, P. Schuster and N. Toro, Dark matter, millicharges, axion and scalar particles, gauge bosons, and other new physics with LDMX, Phys. Rev. D 99 (2019) 075001 [arXiv:1807.01730] [INSPIRE].

[64] ATLAS collaboration, Search for invisible Higgs boson decays with vector boson fusion signatures with the ATLAS detector using an integrated luminosity of $139 \mathrm{fb}^{-1}$, ATLAS-CONF-2020-008 (2020).

[65] A. Berlin, D. Hooper and S.D. McDermott, Simplified dark matter models for the Galactic Center gamma-ray excess, Phys. Rev. D 89 (2014) 115022 [arXiv: 1404.0022] [INSPIRE].

[66] J.L. Feng and J. Smolinsky, Impact of a resonance on thermal targets for invisible dark photon searches, Phys. Rev. D 96 (2017) 095022 [arXiv:1707.03835] [INSPIRE].

[67] B. Li and Y.-F. Zhou, Direct detection of dark matter with resonant annihilation, Commun. Theor. Phys. 64 (2015) 119 [arXiv:1503.08281] [INSPIRE].

[68] D. Tucker-Smith and N. Weiner, Inelastic dark matter, Phys. Rev. D 64 (2001) 043502 [hep-ph/0101138] [INSPIRE].

[69] A. De Simone, V. Sanz and H.P. Sato, Pseudo-Dirac dark matter leaves a trace, Phys. Rev. Lett. 105 (2010) 121802 [arXiv: 1004.1567] [INSPIRE].

[70] R. Krall and M. Reece, Last electroweak WIMP standing: pseudo-Dirac Higgsino status and compact stars as future probes, Chin. Phys. C 42 (2018) 043105 [arXiv: 1705.04843] [INSPIRE].

[71] K. Griest and D. Seckel, Three exceptions in the calculation of relic abundances, Phys. Rev. D 43 (1991) 3191 [INSPIRE]. 